\title{
Controlling Spin-Orbit Interactions in Silicon Quantum Dots Using Magnetic Field Direction
}

\author{
Tuomo Tanttu, ${ }^{1, *}$ Bas Hensen, ${ }^{1}$ Kok Wai Chan, ${ }^{1}$ Chih Hwan Yang,,${ }^{1}$ Wister Wei Huang, ${ }^{1}$ Michael Fogarty, ${ }^{1, \hbar}$ Fay Hudson, ${ }^{1}$ \\ Kohei Itoh, ${ }^{2}$ Dimitrie Culcer, ${ }^{3,4}$ Arne Laucht, ${ }^{1}$ Andrea Morello, ${ }^{1}$ and Andrew Dzurak ${ }^{1, \dagger}$ \\ ${ }^{1}$ Center for Quantum Computation and Communication Technology, School of Electrical Engineering and \\ Telecommunications, The University of New South Wales, Sydney, NSW 2052, Australia \\ ${ }^{2}$ School of Fundamental Science and Technology, Keio University, 3-14-1 Hiyoshi, Kohokuku, \\ Yokohama 223-8522, Japan \\ ${ }^{3}$ School of Physics, The University of New South Wales, Sydney 2052, Australia \\ ${ }^{4}$ ARC Centre for Excellence in Future Low-Energy Electronics Technologies, Sydney 2052, Australia
}

(Received 22 October 2018; revised manuscript received 14 February 2019; published 10 May 2019)

\begin{abstract}
Silicon quantum dots are considered an excellent platform for spin qubits, partly due to their weak spin-orbit interaction. However, the sharp interfaces in the heterostructures induce a small but significant spin-orbit interaction that degrades the performance of the qubits or, when understood and controlled, could be used as a powerful resource. To understand how to control this interaction, we build a detailed profile of the spin-orbit interaction of a silicon metal-oxide-semiconductor double quantum-dot system. We probe the derivative of the Stark shift, $g$-factor and $g$-factor difference for two single-electron quantum-dot qubits as a function of external magnetic field and find that they are dominated by spin-orbit interactions originating from the vector potential, consistent with recent theoretical predictions. Conversely, by populating the double dot with two electrons, we probe the mixing of singlet and spin-polarized triplet states during electron tunneling, which we conclude is dominated by momentum-term spin-orbit interactions that vary from $1.85 \mathrm{MHz}$ up to $27.5 \mathrm{MHz}$ depending on the magnetic field orientation. Finally, we exploit the tunability of the derivative of the Stark shift of one of the dots to reduce its sensitivity to electric noise and observe an $80 \%$ increase in $T_{2}^{*}$. We conclude that the tuning of the spin-orbit interaction will be crucial for scalable quantum computing in silicon and that the optimal setting will depend on the exact mode of qubit operations used.
\end{abstract}

DOI: 10.1103/PhysRevX.9.021028

Subject Areas: Quantum Physics,

Semiconductor Physics, Spintronics

\section{INTRODUCTION}

Silicon-based spin qubits have attracted attention as candidates for large-scale quantum computing thanks to their long coherence times, excellent controllability, and fabrication techniques, which are well established in the semiconductor industry [1-13]. Even though it is weak compared, for instance, to GaAs, the spin-orbit interaction (SOI) significantly affects the behavior of silicon spin

\footnotetext{
* Corresponding author. t.tanttu@unsw.edu.au

Corresponding author. a.dzurak@unsw.edu.au

${ }^{\ddagger}$ Current address: London Centre for Nanotechnology, UCL, 17-19 Gordon Street, London WC1H 0AH, United Kingdom.

Published by the American Physical Society under the terms of the Creative Commons Attribution 4.0 International license. Further distribution of this work must maintain attribution to the author(s) and the published article's title, journal citation, and DOI.
}

qubits, especially through the dependence of the SOI on the valley state $[12,14,15]$. SOI is responsible for effects such as the Stark shift of the electron spin resonance (ESR) frequency, variation of Lande $g$-factors, and mixing between singlet (S) and polarized triplet $\left(\mathrm{T}^{-}\right)$states [15-18]. These effects can be harnessed, for instance, to drive the ESR transition electrically via the Stark shift or by exploiting the variation in the $g$-factors to address qubits individually with a global microwave (MW) field [14,19-21]. In contrast, spin-orbit (SO) effects such as spin-flip tunneling and strong Stark shift can cause state leakage or increased sensitivity to electric noise [14,16,22]. Hence, understanding and controlling the SOI will be important for spin qubit control in larger arrays of dots in the future [23-25].

Here, we fully characterize the SOI and demonstrate how we can tune it in a silicon metal-oxide-semiconductor (SiMOS) double quantum-dot (QD) structure. The structure studied here is shown in Fig. 1(b) and described in Ref. [24]. We vary the direction of the external magnetic field and measure quantities such as the $g$-factor of one dot, the $g$-factor difference between the dots, the Stark effect, 
the dephasing time $T_{2}^{*}$, and $\mathrm{S}-\mathrm{T}^{-}$mixing. The $g$-factor, Stark shift, and $\mathrm{S}^{-\mathrm{T}^{-}}$mixing exhibit sinusoidal dependence on the magnetic field direction, as reported before $[12,15,17,26]$. We use these measurements to extract the Rashba and Dresselhaus interaction strengths of the lowerenergy-valley state in both of the dots. By adjusting the magnetic field direction, the $g$-factor difference can be minimized, allowing global ESR, or it can be maximized in order to address the qubits individually. We employ the tunability of the SOI to reduce the sensitivity of the dot to charge noise and observe an increase of $80 \%$ in $T_{2}^{*}$ near the point where the derivative of the Stark shift vanishes $[15,16]$. Finally, from studying the $\mathrm{S}^{-\mathrm{T}^{-}}$transition, we identify that the coupling in this system is caused by the dynamic spin-orbit field induced by charges moving between the dots, rather than by the hyperfine coupling or the differences in the $g$-tensors between the dots $[18,27]$. This answers a question raised in a previous study [24]. By adjusting the magnetic field direction, we can minimize the mixing between the spin states. This minimization could be extremely useful in reducing errors during spin shuttling of electron spins in a quantum bus, or in reducing undesired leakage to the $\mathrm{T}^{-}$state in the S-T operational basis.

\section{SPIN-ORBIT INTERACTION IN SILICON QUANTUM DOTS}

Three main mechanisms are responsible for SOI in QDs: structural inversion asymmetry (Rashba) [28], bulk inversion asymmetry (Dresselhaus) [29], and interface inversion asymmetry [30]. In bulk silicon, the Dresselhaus term is absent; however, as shown in Ref. [30], interface inversion asymmetry has the same representation in the Hamiltonian as the Dresselhaus term. Hence, to keep the terminology simple, we refer to this as the Dresselhaus term. In a quantum dot, the Rashba interaction leads to a renormalization of the in-plane $g$-factor, while the Dresselhaus interaction gives rise to shear terms in the in-plane Zeeman response, leading to an anisotropy in the $g$-factor [12,14-17]. These effects allow tuning of the SOI by changing the orientation of the external magnetic field. Since at silicon interfaces the Dresselhaus term is expected to be dominant [15-17], we can completely turn off SOI on demand by inducing a Dresselhaus effect that is equally strong but opposite in sign to the Rashba effect [12,15,17,22,31].

In silicon, a SO Hamiltonian for the $i$ th valley can be written as

$H_{\mathrm{SO}, v_{i}}=\alpha_{i}\left(k_{x} \sigma_{y}-k_{y} \sigma_{x}\right)+\beta_{i}\left(k_{x} \sigma_{x}-k_{y} \sigma_{y}\right), \quad i=1,2$,

where $\alpha$ and $\beta$ are the Rashba and Dresselhaus interaction coefficients, $k_{x}$ and $k_{y}$ are the electron wave operators along the [100] and [010] lattice directions, respectively, and $\sigma_{x}$ are $\sigma_{y}$ are the Pauli spin matrices. The electron wave operator is represented as $k_{x}=-i[d /(d x)]+e A_{x} / \hbar$, where $A_{x}$ is the $x$ component of the vector potential of the magnetic field, $e$ is the elementary charge, and $\hbar$ is the reduced Planck's constant. In Ref. [15], it is shown that by choosing a gauge $\vec{A}=\left(B_{y} z,-B_{x} z, 0\right)$ and by averaging over the $z$ axis, we obtain from Eq. (1) corrections to the $g$-tensor. Here, we use two different experimental spin-orbit coefficients to describe the system. We use $\alpha_{g}$ and $\beta_{g}$ for the Rashba and Dresselhaus coefficients that we associate with the vector potential contribution (namely, the $g$-factor and Stark shift). Second, to the dot quantities that are affected by the $[d /(d x)]$ term in $H_{\mathrm{SO}}$ (namely, $\mathrm{S}^{-\mathrm{T}^{-}}$mixing), we associate the coefficients $\alpha_{t}$ and $\beta_{t}$.

The $g$-tensor of a silicon quantum dot at an interface can be expressed as a $3 \times 3$ matrix, and it usually assumes the expression

$$
\hat{g}=\left(\begin{array}{ccc}
g_{\|}-\frac{2 \alpha_{g}^{*}}{\mu_{B}} & \frac{2 \beta_{g}^{*}}{\mu_{B}} & g_{x z} \\
\frac{2 \beta_{g}^{*}}{\mu_{B}} & g_{\|}-\frac{2 \alpha_{g}^{*}}{\mu_{B}} & g_{y z} \\
g_{x z} & g_{y z} & g_{\perp}
\end{array}\right),
$$

where $g_{\|}$is the $g$-factor in plane with the quantum dot, $g_{\perp}$ is the $g$-factor perpendicular to the quantum-dot plane, $\mu_{B}$ is the Bohr magneton, and $\alpha_{g}^{*}=\left[\left(e\left\langle z-z_{i}\right\rangle \alpha_{g}\right) / \hbar\right]$, $\beta_{g}^{*}=\left[\left(e\left\langle z-z_{i}\right\rangle \beta_{g}\right) / \hbar\right]$, with $\left\langle z-z_{i}\right\rangle$ being the spread of the electron wave function in the $z$ direction $\left(z_{i}\right.$ is the location of the interface) $[12,14,15,32]$. Nonzero $g_{x z}$ and $g_{y z}$ terms can be generated from dipole matrix elements [33], for example, from strong in-plane electric fields due to strain caused by thermal expansion mismatch when the device is cooled down [34]. For our quantum dots, we use $\left\langle z-z_{i}\right\rangle=1.68 \mathrm{~nm}$ [14]. In silicon QDs, this tensor is diagonal, and both $g_{\|}$and $g_{\perp}$ are close to the vacuum value of the electron $g$-factor due to the large band gap in silicon [35]. Here, we assume a symmetric $g$-tensor and that the inplane $B_{x}$ coordinate is aligned with the [100] crystal lattice direction. To obtain the $g$-factor along a certain direction, one can use $g=\sqrt{\hat{r}\left(\varphi_{B}, \theta_{B}\right)^{\dagger} \hat{g}_{1}^{\dagger} \hat{g}_{1} \hat{r}\left(\varphi_{B}, \theta_{B}\right)}$, where $\hat{r}\left(\varphi_{B}, \theta_{B}\right)$ is a unit vector pointing in the same direction as the external magnetic field $B$ expressed in Cartesian coordinates.

\section{EXPERIMENTAL $g$-TENSOR}

Figure 1(b) shows the scanning electron microscope (SEM) image of the SiMOS device used in this experiment. This sample was previously used for experiments described in Ref. [24], and the structure is considered a candidate for scaling up to a logical qubit in silicon [23]. One quantum dot is induced at the $\mathrm{Si} / \mathrm{SiO}_{2}$ interface under each of the plunger gates $\mathrm{G} 1$ and $\mathrm{G} 2$, as shown in the schematic cross section in Fig. 1(a). The quantum dots are confined laterally by a confinement barrier (CB) and a barrier gate (BG), which is also used to tune the tunnel rates between the reservoir and the dots. The reservoir is induced under an 
(a)

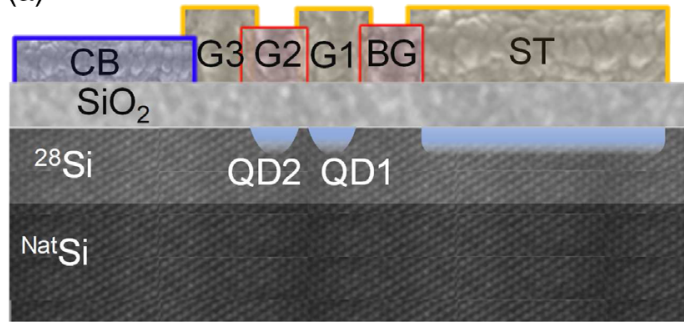

(b)

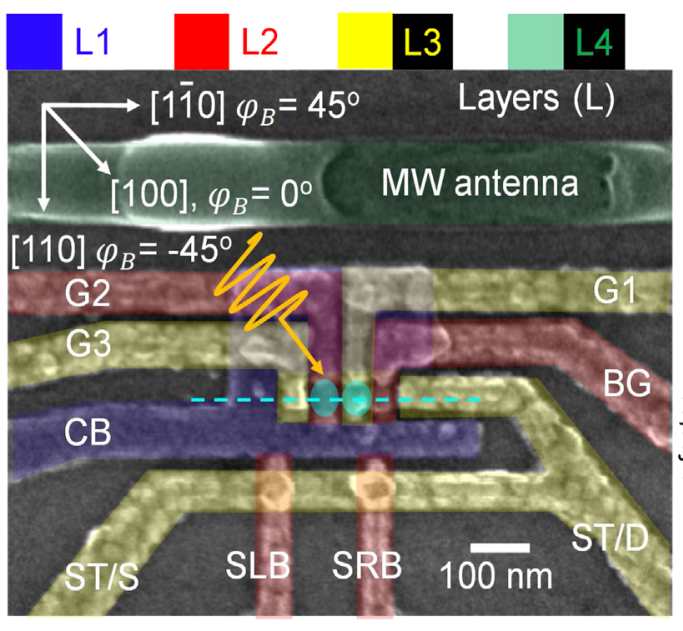

(c) $[\overline{100}[\overline{1} 10][010][110][100][1 \overline{10}][0 \overline{10}][\overline{110}][\overline{100}]$

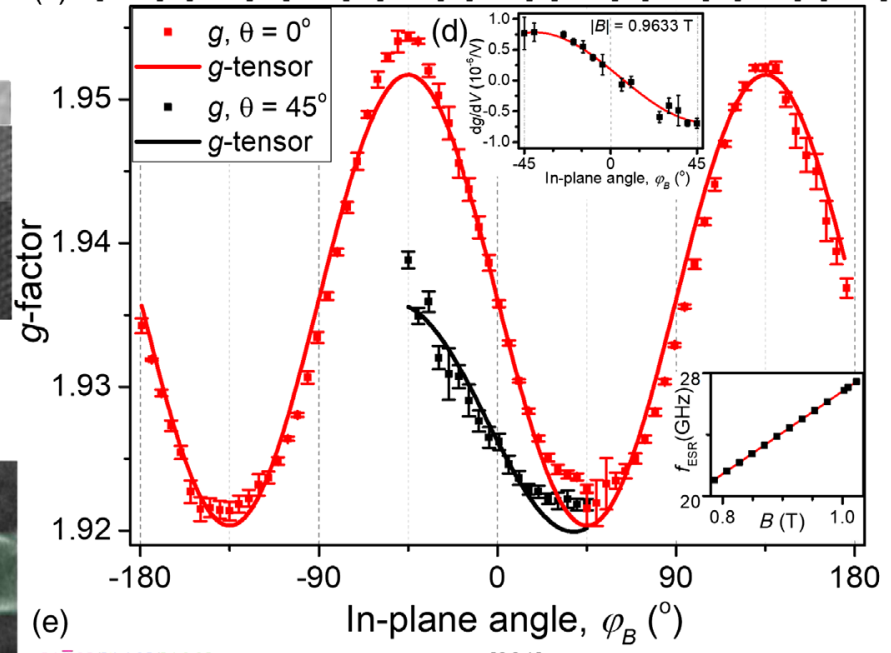

(e)

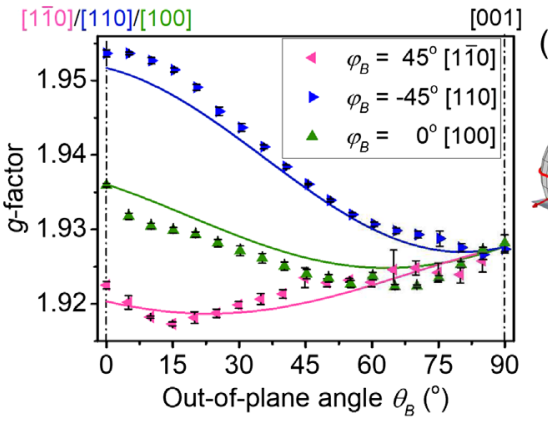

(f)

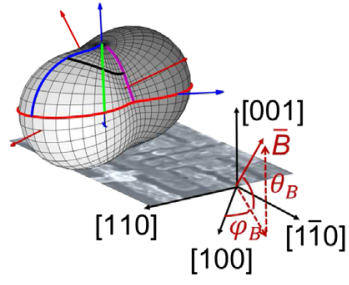

FIG. 1. Sample $g$-factor measurement. (a) Schematic cross section at the position of the dots (QD1 and QD2) along the dotted line in panel (b). (b) False color SEM image of our sample. The MW antenna used to drive the qubits is on the top, the qubit dots are in the middle, and the SET used to sense the dots is at the bottom. (c) $g$-factor of the electron occupying the G1 dot as a function of external magnetic field angle in plane and at $45^{\circ}$ out of plane of the sample in the $(1,0)$ charge configuration. The solid lines correspond to the estimate extracted from the complete $g$-tensor. Inset: Example of ESR frequency as a function of magnetic field with a linear fit that is used to extract the $g$-factor. (d) The derivative of the Stark shift as a function of in-plane magnetic field angle. (e) Out-of-plane $g$-factors measured (colored symbols) with estimates from complete a $g$-tensor (solid lines). (f) Notation of the magnetic field angles with respect to the sample and isosurface of the $g$-factor of the G1 dot based on a single $g$-tensor. We have subtracted 1.9 in order to visualize the anisotropy of the $g$-factor. Blue arrows correspond to laboratory coordinates, and red arrows correspond to the principal axes of the $g$-factor ellipsoid. The data in panels (c) and (e) are taken along the lines shown on the surface.

extension of our sensor top gate (ST). The MOS singleelectron transistor (SET) sensor itself has two gates, left and right (SLB and SRB), that are used to form barriers between the SET leads and the island, which is capacitively coupled to the qubit dots in order to sense changes in the charge state. The MW antenna is used to coherently control the state of the qubit. We note from the image that there is a discontinuity in the MW antenna, likely caused by an electric shock to the device. Despite this discontinuity, we are able to drive the spin transition by applying MW frequency excitation to the antenna. We believe that this spin drive is caused by electrical drive via valley mixing $[19,20]$ or residual magnetic field drive despite the break or combination of both effects [36]. We note that the extracted spin resonance frequency $\left(f_{\mathrm{ESR}}\right)$ does not depend on the driving mechanism.

In Fig. 1(f), we define the magnetic field direction with spherical coordinates so that $\varphi_{B}=0^{\circ}$ corresponds to the
[100] Miller-index direction. This direction is tilted by 45 degrees from the main axis of the sample and the coils of the vector magnet that are aligned along the [110] lattice direction (corresponding to $\varphi_{B}=-45^{\circ}$ ). We also define $\theta_{B}=0^{\circ}$ when the magnetic field is in plane with the sample and $\theta_{B}=90^{\circ}$ when the magnetic field is pointing perpendicular to the sample plane and aligned with [001].

The g-factor of QD1 for a particular external magnetic field direction $\left(\varphi_{B}, \theta_{B}\right)$ is determined in the $(1,0)$ charge configuration by measuring $f_{\mathrm{ESR}}$ as a function of magnetic field amplitude $|B|$. We obtain $g\left(\varphi_{B}, \theta_{B}\right)$ by fitting the linear slope [see inset of Fig. 1(c)] in order to exclude magnetic field hysteresis of the superconducting magnet coils. We show the measurements taken with the magnetic field in plane with the sample in Fig. 1(c), together with measurements taken at 45 degrees out of plane. The out-ofplane data sets are shown in Fig. 1(e). Initialization is performed by loading a spin-down electron, while read-out 
(a)

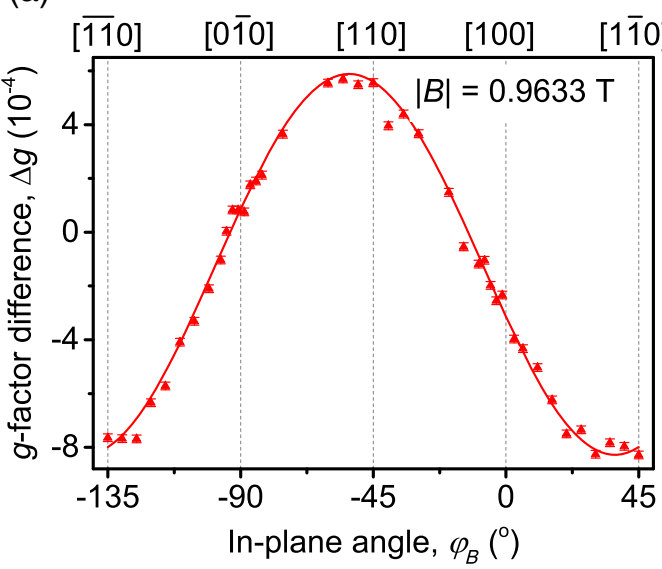

(b)

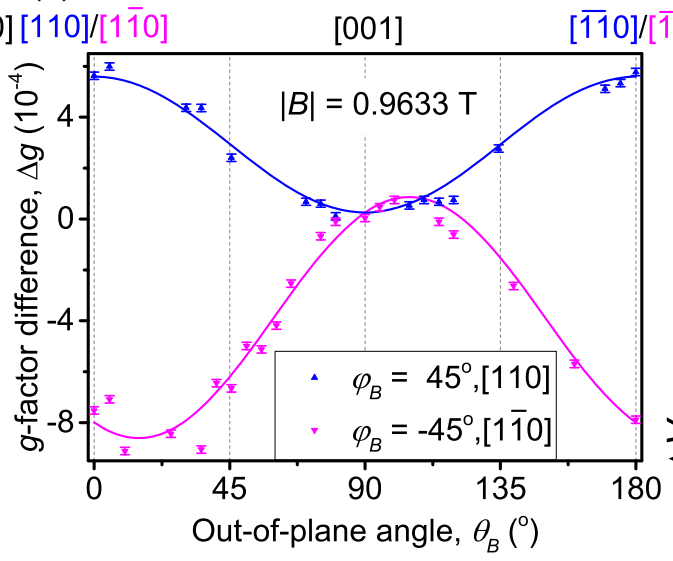

(c)
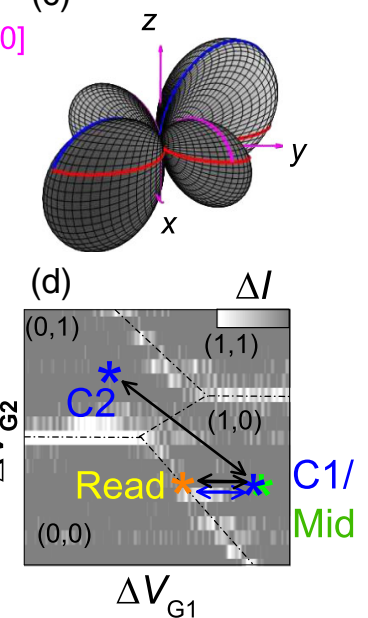

FIG. 2. The $g$-factor difference between the dots. (a) Difference of $g$-factors between the dots under G1 and G2 with occupation (1,0) and $(0,1)$ as a function of in-plane magnetic field angle, together with sinusoidal fit. (b) Difference of $g$-factors between the dots under G1 and G2 as a function of out-of-plane magnetic field angle along [110] (blue) and [110] (magenta). (c) Isosurface representation of the absolute value of $g$-factor difference as a function of magnetic field orientation. The magenta arrows represent crystal lattice orientation. The red, blue, and magenta curves correspond to the fits in panels (a) and (b). (d) Pulse sequences (black and blue arrows) used to measure the $g$-factor difference in panels (a) and (b). $\mathrm{C} 1$ and $\mathrm{C} 2$ indicate the two different control points in the two different sequences.

of the spin state is based on spin-dependent tunneling at the $(0,0) \rightarrow(1,0)$ charge transition, using standard Elzerman read-out [37]. To speed up the measurements, we employ an adiabatic ESR drive [38] to reduce the number of measurement points required to find $f_{\mathrm{ESR}}$ down to $100-\mathrm{kHz}$ accuracy using a constant 500- $\mu$ s ESR pulse.

We use both the out-of-plane and in-plane data to fit a single symmetric $\hat{g}_{1}$ tensor for QD1. This $g$-tensor is

$$
\hat{g}_{1}=1.9 \times I+\left(\begin{array}{ccc}
36.0 & -15.7 & -5.7 \\
-15.7 & 36.0 & -0.3 \\
-5.7 & -0.3 & 28.0
\end{array}\right) \times 10^{-3}
$$

where $I$ is the $3 \times 3$ identity matrix. All the terms above have error bars of $\pm 10^{-3}$, with $95 \%$ confidence intervals. In Fig. 1(f), the full g-factor isosurface is shown (after subtracting a radius of 1.9 to visualize the variation). The solid lines in Figs. 1(c) and 1(e) correspond to $g$-factors reconstructed from the tensor. We note that $g_{x z}$ significantly differs from zero and would correspond to a 0.6-nm dipole matrix element in Ref. [33]. From the tensor, we extract the Dresselhaus term associated with the vector potential $\beta_{g}^{*}=\left|\mu_{B} g_{x y}^{1} / 2\right|=109.9 \pm 6.9 \mathrm{MHz} / \mathrm{T}$, resulting in $\beta_{g}=(178 \pm 11) \times 10^{-13} \mathrm{eV}$ cm for electrons occupying the lower energy valley. We note that due to the observed $\sin 2 \varphi_{B}$ dependence on the $g$-factor, it is unlikely that there is an interface step in the vicinity of the dot since such a step would break this periodicity [14-16].

To extract the Rashba interaction strength, we additionally measure the Stark shift due to the top gate voltage as a function of magnetic field in-plane angle in the same charge configuration [Fig. 1(d)]. Subsequently, we can extract the ratio between Rashba and Dresselhaus coefficients since Rashba corresponds to the offset of the sinusoidal fit $A \sin 2 \varphi_{B}+B$ and Dresselhaus corresponds to the amplitude of the total sine wave [14-16] such that $\left(\alpha_{g} / \beta_{g}\right)=$ $(B / A)=0.0852 \pm 0.0362$. This result is consistent with previous observations of the Dresselhaus effect being stronger than the Rashba effect in MOS dots [17]. The Rashba interaction strength is found to be $\alpha_{g}^{*}=9.36 \pm$ $3.96 \mathrm{MHz} / \mathrm{T}$ and $\alpha_{g}=(15.2 \pm 6.4) \times 10^{-13} \mathrm{eV} \mathrm{cm}$.

\section{THE $g$-FACTOR DIFFERENCE}

In a silicon quantum-dot qubit array, the SOI can vary from one dot to another, which leads to a variety of $g$ factors [14]. This variation in the SOI is caused by differences in the microscopic structure of the quantum dots, such as surface roughness and lattice imperfections [16]. Differences in $g$-factors allow one to individually address the qubits with a global MW field. On the other hand, if the differences vanish, it is possible to drive all qubits with one MW frequency, which is useful for scalable applications [23]. To measure the $g$-factor difference between neighboring dots, we operate near the $(1,0)-(0,1)$ anticrossing and use the pulse sequences depicted in Fig. 2(d). First, the ESR frequency of QD1 is measured by pulsing between the control point $\mathrm{C} 1$ and read-out or initialization. Then, we shuttle the electron through the anticrossing to $\mathrm{C} 2$ where we rotate the spin. Next, we pulse back to $\mathrm{C} 1$ and read out the spin state. This method will determine $f_{\mathrm{ESR}}$ of QD1 and QD2 at the same magnetic field to yield the $g$-factor difference. 
We probe the $g$-factor difference as a function of $\varphi_{B}$ at $\theta_{B}=0$ and as a function of $\theta_{B}$ at $\varphi_{B}=-45^{\circ}, 45^{\circ}$, with the results shown in Figs. 2(a) and 2(b). In the Supplemental Material [39], we use the measured $g$-factor difference and $\hat{g}_{1}$ to estimate the $g$-tensor for the second dot. We can use $\hat{g}_{1}$ and $\hat{g}_{2}$ to determine the difference in the full $\varphi_{B}-\theta_{B}$ space. Figure 2(c) shows the absolute value of the difference as an isosurface. From these measurements, we extract the difference of the Rashba and Dresselhaus interactions between the dots to be $\Delta \alpha_{g}^{*}=2.04 \mathrm{MHz} / \mathrm{T}$ and $\Delta \beta_{g}^{*}=10.07 \mathrm{MHz} / \mathrm{T}$. Notably, similar values for the SOI difference have been previously reported in a MOS double-dot structure [12].

\section{COHERENCE TIME}

The SOI also affects the Stark shift [14-16] and is therefore related to the coherence time $T_{2}^{*}$. This is mainly because in the presence of charge noise, the Stark shift causes the ESR frequency to fluctuate and the phase of the quantum state is lost. To minimize this decoherence, the Dresselhaus effect can be tuned to cancel the Rashba effect at a magic angle of magnetic field, thereby minimizing the SOI. Since SOI is dominated by the Dresselhaus effect, this minimization will happen close to the point where the magnetic field is aligned with the [100] lattice direction $[15,16]$.

In this sample, $T_{2}^{*}$ in the $(1,0)$ charge configuration is too short to be measured reliably, whereas in the $(3,0)$ charge configuration, we measure $T_{2}^{*}$ of around $5 \mu \mathrm{s}$ [14]. We therefore measure the Stark shift at the $(3,0)$ charge configuration as a function of the in-plane magnetic field angle. As shown in Fig. 3(a), the Stark shift vanishes at $\varphi_{B}=-3^{\circ}$, close to the [100] lattice direction. This angle corresponds to a point where the spin-orbit interaction due to the Rashba effect and Dresselhaus effect cancels. In this charge configuration, we have $\left(\alpha_{g} / \beta_{g}\right)=0.041 \pm 0.006$ associated with the upper-energy-valley state. Here, we separate two different noise sources: the decoherence caused by voltage fluctuations $\sigma_{V}$ and other sources. We use a simplified model for $T_{2}^{*}$ that reads

$$
\frac{1}{T_{2}^{*}}=\frac{1}{T_{2 \sigma_{V}}^{*}}+\frac{1}{T_{2 \text { other }}^{*}}
$$

where $T_{2 \sigma_{V}}^{*}$ is the coherence time that is limited by the voltage noise from the top gate and $T_{2 \text { other }}^{*}$ is the coherence time limited by all the other noise sources such as magnetic noise. Now, $T_{2 \sigma_{V}}^{*}$ assumes the expression

$$
T_{2 \sigma_{V}}^{*}=\frac{\sqrt{2} \hbar}{\Delta F_{Z}\left|\frac{d g}{d F_{Z}}\right| \mu_{B} B},
$$

where $\hbar$ is Planck's constant, $\Delta F_{Z}$ is the standard deviation of electric field along the $z$ axis, $\left[(d g) /\left(d F_{Z}\right)\right]$ is the
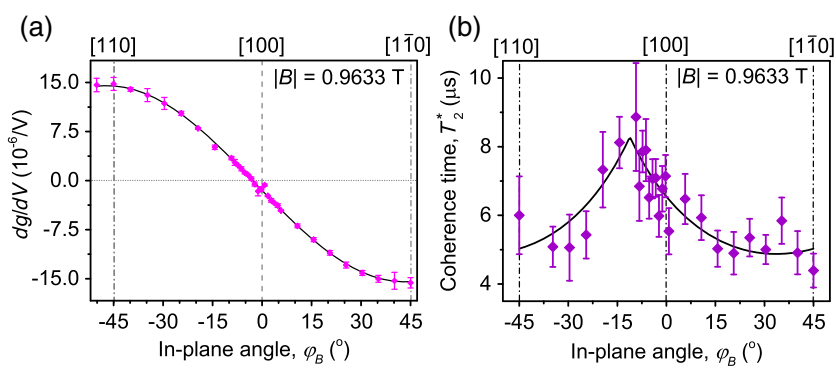

FIG. 3. The derivative of the Stark shift and $T_{2}^{*}$ measurement. (a) Stark shift and (b) decoherence time $T_{2}^{*}$ of the qubit defined by the G1 quantum dot in the $(3,0)$ charge configuration as a function of the external magnetic field in-plane angle $\varphi_{B}$.

derivative of the Stark shift, and $B$ is the strength of the external magnetic field [16]. We assume that the only source of noise is the electrical noise along the $z$ axis. Decoherence caused by electrical noise along the $x$ and $y$ directions is significantly less prominent than along the $z$ axis (see Ref. [39] for details).

We measure $T_{2}^{*}$ by using Ramsey interferometry (see Ref. [39] for details) with integration times of 70 minutes. As seen in Fig. 3(b), the coherence time peaks at $\varphi_{B}=-10^{\circ}$, close to the point where the Stark shift vanishes. The difference could be caused by the fact that we are partially driving the transition electrically, and this causes the Rabi frequency to vary during the measurement. The $T_{2}^{*}$ increases from around $5 \mu \mathrm{s}$ when magnetic field is aligned along [110] up to $8.8 \mu \mathrm{s}$ at the magic angle. From the peak point, we extract $T_{2, \text { other }}^{*}=8.2 \mu \mathrm{s}$ and $T_{2 \sigma_{V},[110]}^{*}=15 \mu \mathrm{s}$. It is worth noting that in a sample where $T_{2, \text { other }}^{*}$ would be longer, the increase of $T_{2}^{*}$ could be significant [16]. In this device, our coherence time is 5-20 times shorter than typically measured in similar samples $[4,40,41]$. There are several possible explanations for this difference. First, it might be due to the partially broken MW antenna, which causes significant electric noise during the drive and induces additional charge noise, reducing the coherence time [36]. Second, despite using isotopically enriched silicon, our decoherence might be limited by residual ${ }^{29} \mathrm{Si}$ nuclei [41], in which case, further isotopic purification is required to reach a point where we are instead limited by the charge noise.

\section{SINGLET AND TRIPLET MIXING DUE TO SPIN-ORBIT INTERACTION}

Avoiding spin flips during tunneling is essential in proposed scalable pathways for silicon spin qubits that rely either on operating in the $\mathrm{S}^{0} \mathrm{~T}^{0}$ basis [23,25] or shuttling of the spins [42]. Two SOI mechanisms that can cause spin flipping are differences in the $g$-tensor offdiagonal terms between the two quantum dots or the induced spin-orbit field due to the interdot tunneling event. The latter spin-orbit effect has previously been studied in 

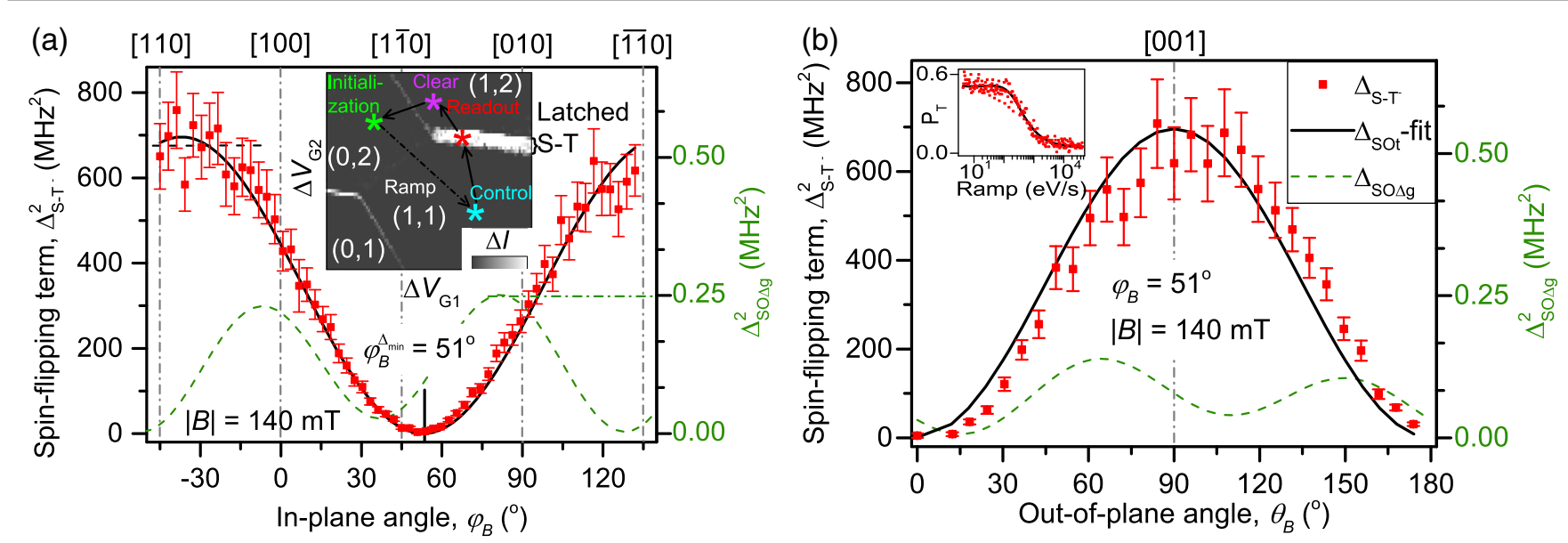

FIG. 4. Singlet-triplet $\mathrm{T}^{-}$mixing as a function of magnetic field direction. (a) Square of the coupling strength of singlet and triplet $\mathrm{T}^{-}$ states as a function of external magnetic field in-plane angle. Inset: Pulse sequence used to measure the coupling. (b) Square of the coupling strength of singlet and triplet $\mathrm{T}^{-}$states as a function of external magnetic field out-of-plane angle while at $\varphi_{B}=51^{\circ}$. We show in (a) and (b) the fit based on the SOI spin-flip model and the estimate from the differences in the off-diagonal terms in the $g$-tensors. Inset: Example fit of the triplet probability as a function of ramp rate across the anticrossing.

double GaAs dots [22,43]. Here, we consider these possible mechanisms that can cause $\mathrm{S}^{-} \mathrm{T}^{-}$mixing and determine their dependence on the external magnetic field direction.

If we choose the magnetic field to be aligned with the $z$ axis, the coupling term between the $\mathrm{S}$ and $\mathrm{T}^{-}$states can be written as $[24,44]$

$$
\Delta_{\mathrm{S}-\mathrm{T}^{-}}(\xi)=\left|\cos (\xi) \frac{\delta E_{Z}^{x}+i \delta E_{Z}^{y}}{\sqrt{2}}+\Delta_{\mathrm{SOt}} \sin (\xi)\right|
$$

where $\delta E_{Z}^{n}$ is the Zeeman energy difference between the dots due to the magnetic field direction $\hat{n}, \Delta_{\text {SOt }}$ is the mixing due to the spin-orbit field caused by the movement of the electron, and $\xi=-\arctan \left[\left(2 t_{c}\right) / E_{Z}\right]$, where $t_{c}$ is the tunnel coupling and $E_{Z}$ the Zeeman energy. The $\delta E_{Z}^{n}$ contributions might arise from differences in the local magnetic fields between the dots or from the differences in the corresponding terms between the $g$-tensors. Differences in the local fields could be induced by an Overhauser field due to the residual ${ }^{29} \mathrm{Si}$ nuclei or Meissner screening [45]. From the previous studies in isotopically enriched ${ }^{28} \mathrm{Si}$, we would expect the contribution from a nuclear spin bath to be random in every direction, with a root mean square of $50 \mathrm{kHz}$ [46]. We exclude the Meissner effect as a possible source of transverse fields since we operate above the critical field of the $\mathrm{Al}$ top gates.

In a previous study, it was found that the mixing between $\mathrm{S}$ and $\mathrm{T}^{-}$states in the same sample was $16.4 \mathrm{MHz}$ [24]significantly higher than expected from the pure hyperfine coupling due to residual ${ }^{29} \mathrm{Si}$ nuclei in the vicinity of the dot [46]. We measure the singlet and triplet $\mathrm{T}^{-}$mixing as a function of magnetic field direction at $140 \mathrm{mT}$, as shown in Figs. 4(a) and 4(b). We operate at the $(1,1)-(2,0)$ anticrossing, with the pulsing scheme presented in the inset of Fig. 4(a), where we use electrically enhanced latched S-T read-out by crossing the $(1,1)-(2,1)$ charge transition [24,47]. We initialize the singlet in the $(2,0)$ charge state and then ramp with a varying ramp rate to $(1,1)$. With decreasing ramp rate, we observe an exponential decrease of the singlet probability seen in the inset of Fig. 4(b). The triplet population due to the ramp across the anticrossing is proportional to $\exp \left(-2 \pi\left|\Delta_{\mathrm{S}^{-} \mathrm{T}^{-}}\right| / \hbar \nu\right)$ [48], where $\left|\Delta_{\mathrm{S}^{-} \mathrm{T}^{-}}\right|$is the mixing between the $\mathrm{S}^{-\mathrm{T}^{-}}$states and $\nu$ is the energy-level ramp rate [24].

The measured dependence of the $\mathrm{S}^{-} \mathrm{T}^{-}$mixing term $\left|\Delta_{\mathrm{S}-\mathrm{T}^{-}}\right|^{2}$ on $\varphi_{B}$ and $\theta_{B}$ is presented in Figs. 4(a) and 4(b). We observe a minimal splitting of $\left|\Delta_{\mathrm{S}^{-} \mathrm{T}^{-}, \min }\right|=$ $1.85 \pm 1 \mathrm{MHz}$ at $\varphi_{B}=51^{\circ}$ in plane and a maximum of $\left|\Delta_{\mathrm{S}-\mathrm{T}^{-}, \max }\right|=27.55 \pm 1.6 \mathrm{MHz}$. We exclude mixing due to the residual ${ }^{29} \mathrm{Si}$ since we observe strong angular dependence of the mixing that is significantly higher than the $50 \mathrm{kHz}$ that we would expect. In Figs. 4(a) and 4(b), we also show the expected angular dependence of $\mathrm{S}^{-\mathrm{T}^{-}}$mixing due to the difference in the off-diagonal elements in the $g$ tensors, $\Delta_{\mathrm{SO} \Delta \mathrm{g}}=\mu_{B} B\left[\left(\delta g^{z x}+i \delta g^{z y}\right) / \sqrt{2}\right]$ (see Ref. [39] for details). This mixing term would exhibit two local maxima and minima in the measurement window with a maximal mixing of $500 \mathrm{kHz}$, which we do not observe. For these reasons, we exclude the difference in the off-diagonal terms in $g$-tensors as a major mechanism for the mixing. In Figs. 4(a) and 4(b), we show the fits based on a model with the spin-orbit field induced by the moving electron. One should note that here, the term $\Delta_{\text {SOt }}$ is associated with interaction strengths $\alpha_{t}$ and $\beta_{t}$ since it arises from the $[d /(d x)]$ term in the Hamiltonian in Eq. (1). This fit is not unique since we have three free parameters $-\alpha_{t}, \beta_{t}$, and the angle of the line of dots with respect to lattice $\delta$-but only 
two numbers to put into the model-amplitude of the mixing and minimum point. In principle, our best guess would be based on the location of the dots based on lithography. As can be seen from Fig. 1(b), the angle for the line of dots with respect to the lattice would correspond to $\delta=45^{\circ}$; however, at this point, the model diverges, and we cannot give a good estimate for the values of spin-orbit coefficients. This model and divergence are discussed in more detail in Ref. [39]. We conclude that the mixing is caused by the induced spin-orbit field due to the movement of the electron observable also in GaAs [22,43]. In a longer array, it is possible that not all dots are aligned on the same line, and hence, there might be no single magnetic field direction where singlet-triplet mixing is minimized. However, appropriate top gate control might be needed to find a single magnetic field that is close to pairwise S-T mixing minimum.

\section{CONCLUSIONS}

We have studied how to control the SOI in a SiMOS double quantum-dot system and used it to improve the performance of the electron spin qubits. This was achieved by probing $g$-factors, the $g$-factor difference, the derivative of the Stark shift, $T_{2}^{*}$, and S-T ${ }^{-}$coupling as a function of the external magnetic field direction. The Rashba and Dresselhaus interaction terms and $g$-tensors of the dots were extracted from the measurements. We also conclude that it is unlikely that there are any interface steps in the vicinity of the dots we are probing. The derivative of the Stark shift, as well as the $g$-factor behavior, is in line with recent theories [15-17], and we observed an increase in $T_{2}^{*}$ near the direction where the derivative of the Stark shift vanishes. We also determined that $\mathrm{S}_{-} \mathrm{T}^{-}$mixing can be explained by the spin-orbit field due to the movement of the charge, and we found the angle for which this mixing is minimized. This result can be used to avoid state leakage to $\mathrm{T}^{-}$when operating in the $\mathrm{S}-\mathrm{T}^{0}$ basis or, in general, to minimize errors when performing spin transport in a long array of dots. In addition, we could enable dynamic nuclear polarization of the residual ${ }^{29} \mathrm{Si}$ by minimizing the mixing due to the SOI, which could otherwise quench the polarization [49].

As shown here, there is a trade-off between addressability and coherence time. When the magnetic field is aligned with [110], where the derivative of the Stark shift is the largest, $T_{2}^{*}$ is the shortest. Our other measurements showed that the difference between the $g$-factors is the largest close to the point where the derivative of the Stark shift is the largest, permitting individual addressability. The corollary is then, if the external field is aligned with [100], where the derivative of the Stark shift should vanish, $T_{2}^{*}$ is longest but we lose the individual addressability. It is also possible that significantly increased $T_{2}^{*}$ (i.e., narrow ESR linewidth) could allow individual addressability if the difference between ESR frequencies is smaller [16]. This idea could be tested with a device that has a significantly longer intrinsic $T_{2}^{*}$.

It is worth noting that in a long array of dots, the $T_{2}^{*}$ in different qubits peaks at slightly different magnetic field directions depending on the individual Rashba and Dresselhaus magnitudes. Since the Rashba interaction is tunable with the top gate voltage, one can align the magnetic field along [100] and tune the addressability by pulsing the gate voltages on demand. Similarly, the $g$ factor differences between qubit pairs will vanish at slightly different points. Hence, choosing an optimal field direction for a long array of dots is not trivial, but it could significantly improve the control fidelity of the qubits in the array. Understanding SOI and its impact on the qubits will be important for scaling up SiMOS qubits into a linear array, or a two-dimensional array for surface-code implementation $[23,25,42]$. The optimal magnetic field direction for a particular choice of qubit operation mode will ultimately require careful weighing of the SOI effects that impact upon key qubit performance parameters, including gate speed, gate fidelities, and state preparation and measurement fidelities. Because of the variation in the SOI and location of the dots, there might not be a single "optimal" magnetic field orientation. For a large array of dots, for instance, we could choose the average of the single or pairwise optimal angle, which would be a significant improvement compared to the magnetic field orientation that is not optimized.

\section{ACKNOWLEDGMENTS}

We thank W. A. Coish, A. Palyi, R. Ruskov, and C. Tahan for inspiring conversations and C. Escott for valuable feedback. We acknowledge support from the US Army Research Office (Grants No. W911NF-13-10024 and No. W911NF-17-1-0198), the Australian Research Council (Grant No. CE170100012), and the NSW Node of the Australian National Fabrication Facility. The views and conclusions contained in this article are those of the authors and should not be interpreted as representing the official policies, either expressed or implied, of the Army Research Office or the U.S. Government. The U.S. Government is authorized to reproduce and distribute reprints for Government purposes notwithstanding any copyright notation herein. B. H. acknowledges support from the Netherlands Organization for Scientific Research (NWO) through a Rubicon Grant. D.C. is supported by the Australian Research Council Centre for Excellence in Future Low-Energy Electronics Technologies (Grant No. CE170100039). K. M. I. acknowledges support from a Grant-in-Aid for Scientific Research by MEXT, NanoQuine, FIRST, and the JSPS Core-to-Core Program.

The authors declare that they have no competing financial interests 


\section{APPENDIX: METHODS}

\section{Device fabrication}

We fabricate our device on an epitaxially grown and isotopically enriched ${ }^{28} \mathrm{Si}$ epilayer that has $800 \mathrm{ppm}$ residue of ${ }^{29} \mathrm{Si}$. Four layers of gates with thicknesses of $25,60,80$, and $80 \mathrm{~nm}$ are fabricated on top of 5.9-nm-thick $\mathrm{SiO}_{2}$ with electron beam lithography and aluminium evaporation. A thermal oxide is grown on top of the $\mathrm{Al}$ gates to isolate layers from each other.

\section{Experimental setup}

The device was bonded to a printed circuit board in a copper enclosure and cooled down in a dilution refrigerator with electron base temperature of $180 \mathrm{mK}$. The dilution refrigerator was equipped with a vector magnet that was calibrated with Hall bars. We had small but noticeable (order of few promille) hysteresis in our superconducting coils, which we observed when calibrating the magnets with Hall bars. In order to avoid the measurement of this hysteresis, we always ramped in a consistent way: We started from 0 field and then ramped the field to $0.5 \mathrm{~T}$ to the corresponding direction and then, with several steps, up to $1 \mathrm{~T}$. We only started measuring ESR frequency after $0.75 \mathrm{~T}$ when the hysteresis was less noticeable. Battery-powered voltage sources were used to provide the dc voltages. Resistive dividers were used to combine the dc voltage and fast gate pulses from the arbitrary waveform generator (Tektronix AWG7122C). At base temperature, the lines for S, D, G1, G2, G3, and B were filtered with a cutoff of $80 \mathrm{MHz}$, and the rest of the gate lines were filtered with a cutoff of $100 \mathrm{~Hz}$. A vector source (Agilent E8267D) was used to generate the MW drive. I/Q modulation of the vector source was used to introduce an adiabatic drive. MWs were attenuated by $10 \mathrm{~dB}$ at $4 \mathrm{~K}$ and $3 \mathrm{~dB}$ at the base plate. Three different stages of adiabatic inversion drive with ranges of $10 \mathrm{MHz}, 1 \mathrm{MHz}$, and $100 \mathrm{kHz}$ were used to narrow down the range for ESR frequency. SET current traces were recorded with a digital oscilloscope and analyzed with a measurement computer.

Elzerman read-out was used to measure the spin occupancy of the dots by pulsing between the control point and the read-out or initialization point that measures the spin states and initializes spin-down [37]. In the singlet-triplet experiment, we used latching read-out in $(1,2)$ charge occupancy, where $\mathrm{T}(1,1)$ are in a metastable blockade [24]. To determine the spin-up or singlet probability, we performed 80-200 single-shot read-outs per point, depending on the measurement.

[1] F. A. Zwanenburg, A. S. Dzurak, A. Morello, M. Y. Simmons, L. C. L. Hollenberg, G. Klimeck, S. Rogge, S. N. Coppersmith, and M. A. Eriksson, Silicon Quantum Electronics, Rev. Mod. Phys. 85, 961 (2013).
[2] J. J. Pla, K. Y. Tan, J. P. Dehollain, W. H. Lim, J. J. L. Morton, D. N. Jamieson, A. S. Dzurak, and A. Morello, A Single-Atom Electron Spin Qubit in Silicon, Nature (London) 489, 541 (2012).

[3] M. Fuechsle, J. A. Miwa, S. Mahapatra, H. Ryu, S. Lee, O. Warschkow, L. C. L. Hollenberg, G. Klimeck, and M. Y. Simmons, A Single-Atom Transistor, Nat. Nanotechnol. 7, 242 (2012).

[4] M. Veldhorst, C. H. Yang, J. C. C. Hwang, W. Huang, J. P. Dehollain, J. T. Muhonen, S. Simmons, A. Laucht, F. E. Hudson, K. M. Itoh, A. Morello, and A. S. Dzurak, A TwoQubit Logic Gate in Silicon, Nature (London) 526, 410 (2015).

[5] A. Bienfait, J. J. Pla, Y. Kubo, M. Stern, X. Zhou, C. C. Lo, C. D. Weis, T. Schenkel, M. L. W. Thewalt, D. Vion, D. Esteve, B. Julsgaard, K. Mølmer, J. J. L. Morton, and P. Bertet, Reaching the Quantum Limit of Sensitivity in Electron Spin Resonance, Nat. Nanotechnol. 11, 253 (2016).

[6] X. Mi, J. V. Cady, D. M. Zajac, P. W. Deelman, and J. R. Petta, Strong Coupling of a Single Electron in Silicon to a Microwave Photon, Science 355, 156 (2016).

[7] R. Maurand, X. Jehl, D. Kotekar-Patil, A. Corna, H. Bohuslavskyi, R. Laviéville, L. Hutin, S. Barraud, M. Vinet, M. Sanquer, and S. De Franceschi, A CMOS Silicon Spin Qubit, Nat. Commun. 7, 13575 (2016).

[8] T. Fujita, T. A. Baart, C. Reichl, W. Wegscheider, and L. M. K. Vandersypen, Coherent Shuttle of Electron-Spin States, npj Quantum Inf. 3, 22 (2017).

[9] N. Samkharadze, G. Zheng, N. Kalhor, D. Brousse, A. Sammak, U.C. Mendes, A. Blais, G. Scappucci, and L. M. K. Vandersypen, Strong Spin-Photon Coupling in Silicon, Science 359 (2018).

[10] T. F. Watson, S. G. J. Philips, E. Kawakami, D. R. Ward, P. Scarlino, M. Veldhorst, D. E. Savage, M. G. Lagally, M. Friesen, S. N. Coppersmith, M. A. Eriksson, and L. M. K. Vandersypen, A Programmable Two-Qubit Quantum Processor in Silicon, Nature (London) 555, 633 (2018).

[11] D. M. Zajac, A. J. Sigillito, M. Russ, F. Borjans, J. M. Taylor, G. Burkard, and J. R. Petta, Resonantly Driven CNOT Gate for Electron Spins, Science 359, 439 (2018).

[12] R. M. Jock, N. T. Jacobson, P. Harvey-Collard, A. M. Mounce, V. Srinivasa, D. R. Ward, J. Anderson, R. Manginell, J. R. Wendt, M. Rudolph, T. Pluym, J. K. Gamble, A. D. Baczewski, W. M. Witzel, and M. S. Carroll, A Silicon MetalOxide-Semiconductor Electron Spin-Orbit Qubit, Nat. Commun. 9, 1768 (2018).

[13] T. Nakajima, M. R. Delbecq, T. Otsuka, S. Amaha, J. Yoneda, A. Noiri, K. Takeda, G. Allison, A. Ludwig, A. D. Wieck, X. Hu, F. Nori, and S. Tarucha, Coherent Transfer of Electron Spin Correlations Assisted by Dephasing Noise, Nat. Commun. 9, 2133 (2018).

[14] M. Veldhorst, R. Ruskov, C. H. Yang, J. C. C. Hwang, F. E. Hudson, M. E. Flatté, C. Tahan, K. M. Itoh, A. Morello, and A. S. Dzurak, Spin-Orbit Coupling and Operation of Multivalley Spin Qubits, Phys. Rev. B 92, 201401(R) (2015).

[15] R. Ruskov, M. Veldhorst, A. S. Dzurak, and C. Tahan, Electron g-factor of Valley States in Realistic Silicon Quantum Dots, Phys. Rev. B 98, 245424 (2018).

[16] R. Ferdous, K. W. Chan, M. Veldhorst, J. C. C. Hwang, C. H. Yang, H. Sahasrabudhe, G. Klimeck, A. Morello, 
A. S. Dzurak, and R. Rahman, Interface-Induced Spin-Orbit Interaction in Silicon Quantum Dots and Prospects for Scalability, Phys. Rev. B 97, 241401(R) (2018).

[17] R. Ferdous, E. Kawakami, P. Scarlino, M. P. Nowak, D. R. Ward, D. E. Savage, M. G. Lagally, S. N. Coppersmith, M. Friesen, M. A. Eriksson, L. M. K. Vandersypen, and R. Rahman, Valley Dependent Anisotropic Spin Splitting in Silicon Quantum Dots, npj Quantum Inf. 4, 26 (2018).

[18] D. Stepanenko, M. Rudner, B. I. Halperin, and D. Loss, Singlet-Triplet Splitting in Double Quantum Dots Due to Spin-Orbit and Hyperfine Interactions, Phys. Rev. B 85, 075416 (2012).

[19] A. Corna, L. Bourdet, R. Maurand, A. Crippa, D. KotekarPatil, H. Bohuslavskyi, R. Laviéville, L. Hutin, S. Barraud, X. Jehl, M. Vinet, S. De Franceschi, Y.-M. Niquet, and M. Sanquer, Electrically Driven Electron Spin Resonance Mediated by Spin-Valley-Orbit Coupling in a Silicon Quantum Dot, npj Quantum Inf. 4, 6 (2018).

[20] W. Huang, M. Veldhorst, N. M. Zimmerman, A. S. Dzurak, and D. Culcer, Electrically Driven Spin Qubit Based on Valley Mixing, Phys. Rev. B 95, 075403 (2017).

[21] W. Huang, C. H. Yang, K. W. Chan, T. Tanttu, B. Hensen, R. C. C. Leon, M. A. Fogarty, J. C. C. Hwang, F. E. Hudson, K. M. Itoh, A. Morello, A. Laucht, and A. S. Dzurak, Fidelity Benchmarks for Two-Qubit Gates in Silicon, arXiv:1805.05027.

[22] A. Hofmann, V. F. Maisi, T. Krähenmann, C. Reichl, W. Wegscheider, K. Ensslin, and T. Ihn, Anisotropy and Suppression of Spin-Orbit Interaction in a GaAs Double Quantum Dot, Phys. Rev. Lett. 119, 176807 (2017).

[23] C. Jones, M. A. Fogarty, A. Morello, M. F. Gyure, A. S. Dzurak, and T. D. Ladd, Logical Qubit in a Linear Array of Semiconductor Quantum Dots, Phys. Rev. X 8, 021058 (2018).

[24] M. A. Fogarty, K. W. Chan, B. Hensen, W. Huang, T. Tanttu, C. H. Yang, A. Laucht, M. Veldhorst, F. E. Hudson, K. M. Itoh, D. Culcer, T. D. Ladd, A. Morello, and A. S. Dzurak, Integrated Silicon Qubit Platform with Single-Spin Addressability, Exchange Control and Single-Shot Singlet-Triplet Readout, Nat. Commun. 9, 4370 (2018).

[25] M. Veldhorst, H. G. J. Eenink, C. H. Yang, and A. S. Dzurak, Silicon CMOS Architecture for a Spin-Based Quantum Computer, Nat. Commun. 8, 1766 (2017).

[26] M. Brauns, J. Ridderbos, A. Li, E. P. A. M. Bakkers, and F. A. Zwanenburg, Electric-Field Dependent g-factor Anisotropy in Ge-Si Core-Shell Nanowire Quantum Dots, Phys. Rev. B 93, 121408(R) (2016).

[27] D. Stepanenko, N. E. Bonesteel, D. P. DiVincenzo, G. Burkard, and D. Loss, Spin-Orbit Coupling and TimeReversal Symmetry in Quantum Gates, Phys. Rev. B 68, 115306 (2003).

[28] E. Rashba, Properties of Semiconductors with an Extremum Loop. 1. Cyclotron and Combinational Resonance in a Magnetic Field Perpendicular to the Plane of the Loop, Sov. Phys. Solid State 2, 1109 (1960).

[29] G. Dresselhaus, Spin-Orbit Coupling Effects in Zinc Blende Structures, Phys. Rev. 100, 580 (1955).

[30] L.E. Golub and E. L. Ivchenko, Spin Splitting in Symmetrical SiGe Quantum Wells, Phys. Rev. B 69, 115333 (2004).
[31] M. O. Nestoklon, E. L. Ivchenko, J.-M. Jancu, and P. Voisin, Electric Field Effect on Electron Spin Splitting in SiGeSi Quantum Wells, Phys. Rev. B 77, 155328 (2008).

[32] A. Crippa, R. Maurand, L. Bourdet, D. Kotekar-Patil, A. Amisse, X. Jehl, M. Sanquer, R. Laviéville, H. Bohuslavskyi, L. Hutin, S. Barraud, M. Vinet, Y.-M. Niquet, and S. De Franceschi, Electrical Spin Driving by g-matrix Modulation in Spin-Orbit Qubits, Phys. Rev. Lett. 120, 137702 (2018).

[33] C. H. Yang, A. Rossi, R. Ruskov, N. S. Lai, F. A. Mohiyaddin, S. Lee, C. Tahan, G. Klimeck, A. Morello, and A.S. Dzurak, Spin-Valley Lifetimes in a Silicon Quantum Dot with Tunable Valley Splitting, Nat. Commun. 4, 2069 (2013).

[34] J. J. Pla, A. Bienfait, G. Pica, J. Mansir, F. A. Mohiyaddin, Z. Zeng, Y. M. Niquet, A. Morello, T. Schenkel, J. J. L. Morton, and P. Bertet, Strain-Induced Spin-Resonance Shifts in Silicon Devices, Phys. Rev. Applied 9, 044014 (2018).

[35] L. M. Roth, g Factor and Donor Spin-Lattice Relaxation for Electrons in Germanium and Silicon, Phys. Rev. 118, 1534 (1960).

[36] J. T. Muhonen, J. P. Dehollain, A. Laucht, F. E. Hudson, R. Kalra, T. Sekiguchi, K. M. Itoh, D. N. Jamieson, J. C. McCallum, A. S. Dzurak, and A. Morello, Storing Quantum Information for 30 Seconds in a Nanoelectronic Device, Nat. Nanotechnol. 9, 986 (2014).

[37] J. M. Elzerman, R. Hanson, L. H. W. van Beveren, B. Witkamp, L. M. K. Vandersypen, and L. P. Kouwenhoven, Single-Shot Read-Out of an Individual Electron Spin in a Quantum Dot, Nature (London) 430, 431 (2004).

[38] G. Feher, Observation of Nuclear Magnetic Resonances via the Electron Spin Resonance Line, Phys. Rev. 103, 834 (1956).

[39] See Supplemental Material at http://link.aps.org/ supplemental/10.1103/PhysRevX.9.021028 for the stability map of the device, g-tensor of the second dot, the analysis of the coherence time, theoretical study of the $\mathrm{S}-\mathrm{T}^{-}$mixing due to the spin-orbit interaction.

[40] M. Veldhorst, J. C. C. Hwang, C. H. Yang, A. W. Leenstra, B. de Ronde, J. P. Dehollain, J. T. Muhonen, F. E. Hudson, K. M. Itoh, A. Morello, and A. S. Dzurak, An Addressable Quantum Dot Qubit with Fault-Tolerant Control-Fidelity, Nat. Nanotechnol. 9, 981 (2014).

[41] K. W. Chan, W. Huang, C. H. Yang, J. C. C. Hwang, B. Hensen, T. Tanttu, F. E. Hudson, K. M. Itoh, A. Laucht, A. Morello, and A. S. Dzurak, Assessment of a Silicon Quantum Dot Spin Qubit Environment via Noise Spectroscopy, Phys. Rev. Applied 10, 044017 (2018).

[42] J. M. Taylor, H.-A. Engel, W. Dür, A. Yacoby, C. M. Marcus, P. Zoller, and M. D. Lukin, Fault-Tolerant Architecture for Quantum Computation Using Electrically Controlled Semiconductor Spins, Nat. Phys. 1, 177 (2005).

[43] P. Scarlino, E. Kawakami, P. Stano, M. Shafiei, C. Reichl, W. Wegscheider, and L. M. K. Vandersypen, Spin-Relaxation Anisotropy in a GaAs Quantum Dot, Phys. Rev. Lett. 113, 256802 (2014).

[44] J. M. Taylor, J. R. Petta, A. C. Johnson, A. Yacoby, C. M. Marcus, and M. D. Lukin, Relaxation, Dephasing, and Quantum Control of Electron Spins in Double Quantum Dots, Phys. Rev. B 76, 035315 (2007). 
[45] D. Underwood, http://meetings.aps.org/link/BAPS.2017 .MAR.F52.8 .

[46] K. Eng, T. D. Ladd, A. Smith, M. G. Borselli, A. A. Kiselev, B. H. Fong, K. S. Holabird, T. M. Hazard, B. Huang, P. W. Deelman, I. Milosavljevic, A. E. Schmitz, R. S. Ross, M. F. Gyure, and A. T. Hunter, Isotopically Enhanced TripleQuantum-Dot Qubit, Sci. Adv. 1, e1500214 (2015).

[47] P. Harvey-Collard, B. D’ Anjou, M. Rudolph, N. T. Jacobson, J. Dominguez, G. A. T. Eyck, J. R. Wendt, T. Pluym, M. P. Lilly, W. A. Coish, M. Pioro-Ladrière, and M. S.
Carroll, High-Fidelity Single-Shot Readout for a Spin Qubit via an Enhanced Latching Mechanism, Phys. Rev. X 8, 021046 (2018).

[48] S. N. Shevchenko, S. Ashhab, and F. Nori, Landau-ZenerStueckelberg Interferometry, Phys. Rep. 492, 1 (2010).

[49] J. M. Nichol, S. P. Harvey, M. D. Shulman, A. Pal, V. Umansky, E. I. Rashba, B. I. Halperin, and A. Yacoby, Quenching of Dynamic Nuclear Polarization by Spin-Orbit Coupling in GaAs Quantum Dots, Nat. Commun. 6, 7682 (2015). 